\title{
1-5 Acquiring a Radiance Distribution to Superimpose Virtual Objects onto a Real Scene
}

\author{
Imari Sato Yoichi Sato Katsushi Ikeuchi \\ Institute of Industrial Science \\ The University of Tokyo *
}

\begin{abstract}
This paper describes a new method for superimposing virtual objects onto an image of a real scene with correct shading and shadows. Unlike the previously proposed methods, our method renders virtual objects based on a real illumination distribution. First, a radiance distribution in the real scene is determined from two omni-directional images of the scene. Then the measured radiance distribution is used for rendering virtual objects superimposed onto the scene image. The proposed method can synthesize a convincing image even for a complex radiance distribution where other methods often fail. We successfully tested the proposed method by using real images to demonstrate its effectiveness.
\end{abstract}

\section{Introduction}

For superimposing virtual objects, i.e., computergenerated objects, onto a real scene appropriately, the following three aspects have to be taken into account: geometry, illumination, and time. More specifically, the virtual object has to be located at a desired location in the real scene, and the object must appear at the correct location in the image (consistency of geometry). Also, shading of the virtual object has to match that of other objects in the scene, and the virtual object must cast a correct shadow, i.e., a shadow whose characteristics are consistent with those of shadows in the real scene (consistency of illumination). Lastly, motions of the virtual object and the real objects have to be coordinated (consistency of time).

In the past, consistency of geometry and consistency of time have been intensively investigated in the field of augmented reality ${ }^{1}$. On the other hand, in a typical augmented reality system, a very simple and ad-hoc illumination model is used for rendering virtual objects and for creating shadows cast by the

Address: 7-22-1 Roppongi, Minato-ku, Tokyo 106-8558 Japan. E-mail: imarik, ysato, ki@̂cvl.iis.u-tokyo.ac.jp [1] virtual objects. This is mainly because real scenes usually include both direct and indirect illumination distributed in a complex way. Therefore, it is not easy to obtain correct illumination models to be used for augmented reality systems.

Previously proposed methods $[5,3]$ computed global illumination using pixel values of an input image and thus cannot model a direct illumination from outside of the input image unless a user specifies the positions of all lights. Moreover, the previous methods required a great deal of manual processes such as specifying object shapes and line segments.

In this paper, we propose a new method for superimposing virtual objects onto a real scene using a radiance distribution of the real scene ${ }^{2}$. First, the proposed method determines a radiance distribution in the real scene by using two omni-directional images of the scene. Then the measured radiance distribution is used for rendering virtual objects superimposed onto the scene image. Compared with the previously proposed methods, our method can measure a radiance distribution from all directions automatically by using a pair of omni-directional images taken by a CCD camera with a fisheye lens.

This paper is organized as follows. Section 2 describes an omni-directional stereo algorithm for measuring a radiance distribution of the real scene. Section 3 explains how to superimpose virtual objects onto the real scene by using the measured radiance distribution. Section 4 shows experimental results of the proposed method applied to real images of both indoor and outdoor environments. Section 5 presents concluding remarks.

\section{Measuring Illumination Distribu- tion(by Omni-Directional Stereo)}

In this section, we explain how to measure a real illumination distribution from a pair of omnidirectional images taken by a CCD camera with a

\footnotetext{
2 Recently, a similar approach was independently developed by Debevec, and the work was reported in [2]
} 

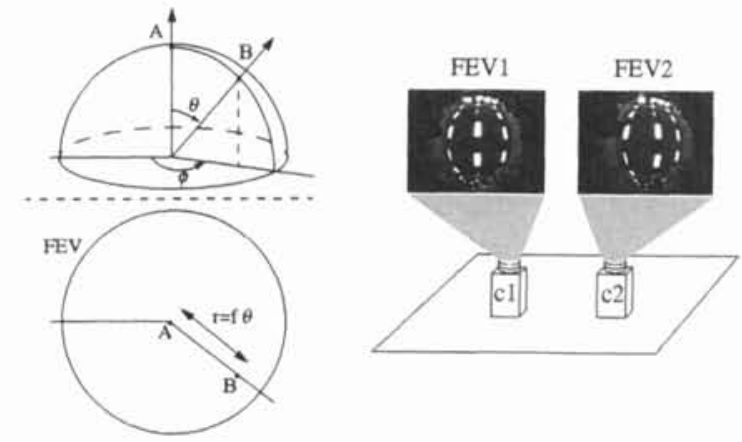

Figure 1: omni-directional image acquisition system

fisheye lens ${ }^{3}$. An acquisition system for the omnidirectional images is illustrated in Figure 1.

First, we extract feature points with high contrast in the two omni-directional images by using the feature extraction algorithm proposed by Tomasi and Kanade [9]. In the algorithm, an image pixel with high gradient values in two orthogonal directions, e.g., a corner point, is extracted as a feature point. Most of incoming light energy in a real scene comes from direct light sources such as a fluorescent lamp and $\mathrm{a}$ window to the outside, while the rest of the incoming light energy comes from indirect illumination such as reflection from a wall. Thus it is important to know the accurate locations of direct light sources to represent an illumination distribution of a real scene. Fortunately, direct light sources usually appear as significantly bright points in an omnidirectional image. Therefore, it should be relatively easy to identify direct light sources in the image.

Secondly, 3D coordinates of points in the real scene corresponding to the extracted feature points are determined by using a stereo algorithm [4]. Then $3 \mathrm{D}$ coordinates of the remaining part of the real scene are approximated by generating a 3D triangular mesh based on the $3 \mathrm{D}$ coordinates of the distinct feature points. This part includes a wall, a ceiling, and other object surfaces that act as indirect light sources and therefore do not appear as a distinct point in omnidirectional images. The obtained triangular mesh approximates an entire shape of the real scene, e.g., the ceiling and walls of a room, which acts as direct or indirect light sources.

Finally, the radiance of the scene is estimated by using the brightness of the omni-directional images. In our report [8], we have shown that the irradiance $E$ on the imaging plane and the radiance $L$ on the object surface in the scene are related as

\footnotetext{
${ }^{3} 3 \mathrm{CCD}$ color camera (Victor KYF-57) and fisheye lens (Fit Corporation FI-19 with field of view of 180 degrees)
}

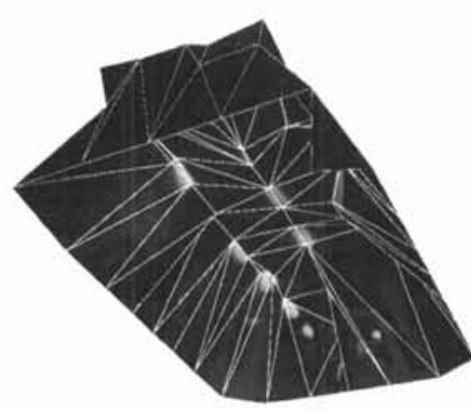

Figure 2: measured radiance distribution

$$
E=L \frac{\pi}{4}\left(\frac{d}{f}\right)^{2}\left(1+\alpha^{2}\right)^{2} \cos ^{3}\left(\tan ^{-1} \alpha\right) \cos \alpha
$$

where $f$ is the focal length, $d$ is the diameter of the fisheye lens and $\alpha$ is the angle between the lens optical axis and the ray from the object surface to the lens center. Therefore, by projecting the omnidirectional image onto the obtained $3 \mathrm{D}$ triangular mesh and by converting the projected color values according to Equation(1), we can obtain the radiance distribution of the scene up to scale.

An example of measured radiance distribution is shown in Figure 2. Note that a real scene contains a very wide range of radiance. Due to the limited dynamic range of a CCD camera, pixel values of an image taken with one shutter speed cannot measure radiance in the scene accurately. To avoid this problem, multiple images taken with different shutter speeds are combined to produce each omni-directional image with a virtually extended dynamic range.

\section{Superimposing Virtual Objects Us- ing Measured Illumination}

After the radiance distribution of the scene is measured, we can render a virtual object and generate shadows cast by the virtual object onto the real scene by using the measured radiance distribution. This can be done in the following steps.

1. For each pixel in the input image of the real scene, a ray extending from the camera projection center through the pixel is generated using the transformation between $3 \mathrm{D}$ world coordinate system and $2 \mathrm{D}$ image coordinate system ${ }^{4}$. Then it is determined whether a ray intersects a virtual object or a real object in the scene.

${ }^{4}$ The camera calibration algorithm proposed by Tsai [11] is used to estimate camera parameters 
2. If the ray intersects a virtual object, we compute a color to be observed at the surface point using the estimated illumination distribution of the real scene. In our method, a simplified TorranceSparrow reflection model $[7,10,6]$ is used as

$$
\begin{array}{r}
I_{c}=K_{d, c} \int_{-\pi}^{\pi} \int_{0}^{\frac{\pi}{2}} L_{c}\left(\theta_{i}, \phi_{i}\right) \cos \theta_{i} \sin \theta_{i} d \theta_{i} d \phi_{i}+ \\
K_{s, c} \int_{-\pi}^{\pi} \int_{0}^{\frac{\pi}{2}} L_{c}\left(\theta_{i}, \phi_{i}\right) \frac{1}{\cos \theta_{r}} e^{\frac{-\gamma\left(\theta_{i}, \phi_{i}\right)^{2}}{2 \sigma^{2}}} \sin \theta_{i} d \theta_{i} d \phi_{i} \\
\mathrm{c}=\mathrm{R}, \mathrm{G}, \mathrm{B}
\end{array}
$$

\section{Experimental Results}

where $\theta_{i}$ is the polar angle, $\phi_{i}$ is the azimuth angle as shown in Figure 1. $L\left(\theta_{i}, \phi_{i}\right)$ is the radiance per unit solid angle coming from the direction $\left(\theta_{i}, \phi_{i}\right), \theta_{r}$ is the angle between the surface normal and the viewing direction, $\gamma\left(\theta_{i}, \phi_{i}\right)$ is the angle between the surface normal and the bisector of the light source direction and the viewing direction, and $K_{d, c}$ and $K_{s, c}$ are constants for the diffuse and specular reflection components, and $\sigma$ is the standard deviation of a facet slope of the Torrance-Sparrow reflection model.

3. Otherwise, we consider that the pixel corresponds to a point on a real object surface. Then we modify an observed color at the point on the real object surface, so that a shadow cast by the virtual object onto the real object is generated.

(1) Obtain a 3D coordinate of a surface point where a ray through an image pixel intersects a surface of a real object in the scene. In this work, we consider only the surface on which a virtual object is placed. The location of the surface is determined from camera calibration.

(2) Compute a total irradiance $E_{1}$ at the surface point using the estimated illumination distribution. In this case, a virtual object does not occlude any incoming lights.

$$
\begin{array}{r}
E_{1}, c=\int_{-\pi}^{\pi} \int_{0}^{\frac{\pi}{2}} L_{c}\left(\theta_{i}, \phi_{i}\right) \cos \theta_{i} \sin \theta_{i} d \theta_{i} d \phi_{i} \\
\mathrm{c}=\mathrm{R}, \mathrm{G}, \mathrm{B}
\end{array}
$$

(3) Compute a total irradiance $E_{2}$ at the surface point in the case where the virtual object occludes some of the incoming lights.

$$
E_{2, c}=\int_{-\pi}^{\pi} \int_{0}^{\frac{\pi}{2}} L_{c}\left(\theta_{1}, \phi_{i}\right) S\left(\theta_{i}, \phi_{i}\right) \cos \theta_{i} \sin \theta_{i} d \theta_{i} d \phi_{i}
$$

$$
c=R, G, B
$$

where $S\left(\theta_{i}, \phi_{i}\right)$ are occlusion coefficients; $S_{i}=0$ if $L\left(\theta_{i}, \phi_{i}\right)$ is occluded by the object; Otherwise $S_{i}=1$.
(4)Compute the ratio of $E_{2}$ to $E_{1}$. The ratio represents how much of the irradiance at the intersection would be lost if the virtual object were placed in the scene. Therefore, by multiplying the ratio $E_{2} / E_{1}$ to the observed color of the image pixel $I$, we obtain the color $I^{\prime}$ that would be the color of the image pixel if there were a virtual object.

$$
I_{c}^{\prime}=I_{\mathrm{c}} \frac{E_{2, c}}{E_{1, c}} \quad c=R, G, B
$$

We have tested the proposed method by using real images taken in both indoor and outdoor environments. The input images used for indoor experiment are shown in Figure 3 and the synthesized results are shown in Figure 4. In the result images, shading of the virtual object blends well into the scene. Also, the virtual object casts a shadow with a soft edge on the tabletop in the same way as the other objects in the scene.

We also applied our method to real images taken in an outdoor environment. The input images used in this experiment are shown in Figure 5 and the superimposed results are shown in Figure 6. The virtual objects that we used in this experiment are a shiny metallic sphere, a rough metallic dodecahedron, and a yellow matte cube, all of which are floating at the same location in the outdoor scene. We can see that shadings and shadows are represented appropriately in the synthesized images by using our method. In particular. the reflection of the surrounding buildings appears appropriately on the metallic sphere. Unless the real radiance distribution of the scene is used as in our method, a convincing reflection on a virtual object as shown in this figure cannot be created.

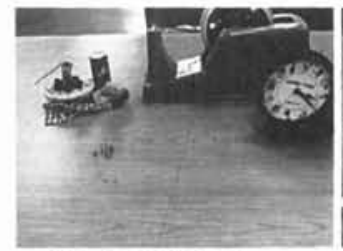

(a) input image

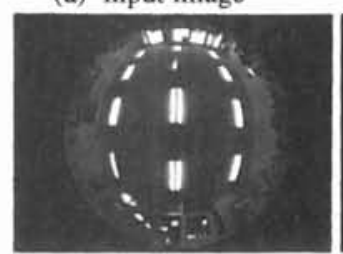

(c) FEV1

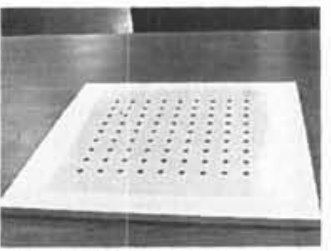

(b) calibration image

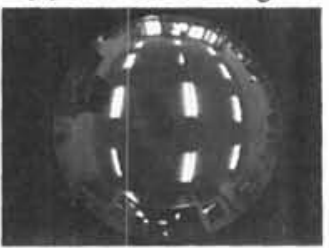

FEV2
Figure 3: (a) input image (b) calibration image (c) omni-directional images 

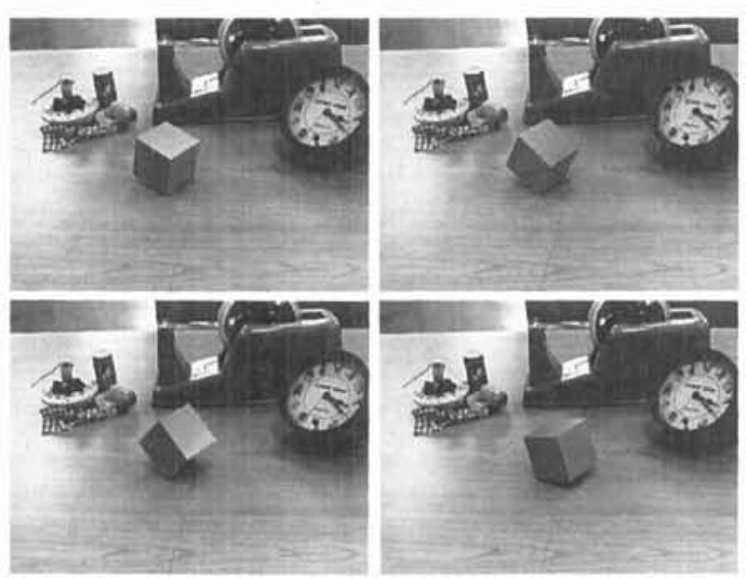

Figure 4: images synthesized with our method

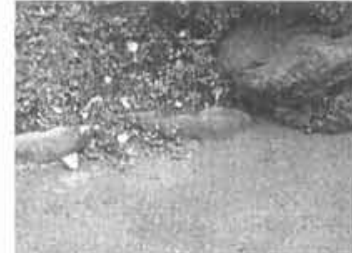

(a) input image

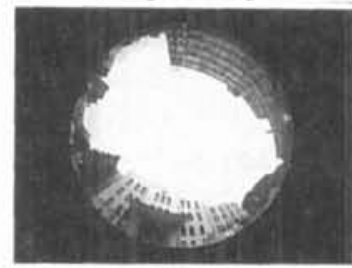

(c) FEV1

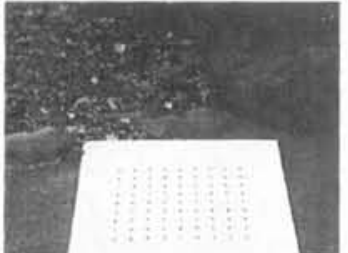

(b) calibration image

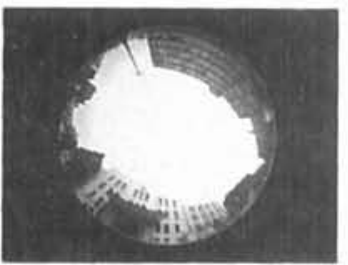

FEV2
Figure 5: (a) input image (b) calibration image (c) omni-directional images
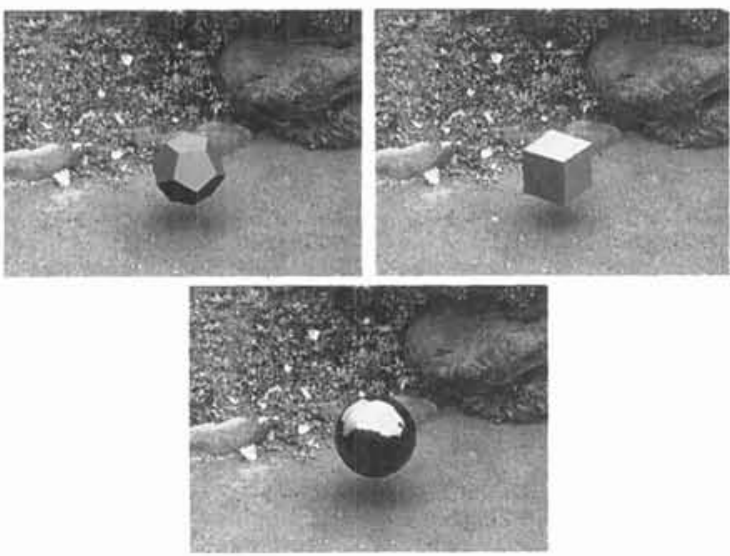

Figure 6: images synthesized with our method

\section{Conclusions}

In this paper, we proposed a new method for superimposing virtual objects onto an image of a real scene by taking into account the radiance distribution of the scene. For matching illumination, the radiance distribution of the real scene is measured by using two omni-directional images of the scene. Unlike the previously proposed methods that use only light sources in an input image of a real scene, our method can measure an entire radiance distribution of the scene by using multiple omni-directional images.

As a result, our method can superimpose virtual objects with convincing shadings and shadows onto the real scene. In addition, we obtain the radiance distribution as a triangular mesh representing the approximate shape of the scene. Therefore, a correct radiance distribution can be used for rendering virtual objects and generating shadows cast by the virtual objects wherever the objects are placed in the real scene.

\section{References}

[1] R. T. Azuma, "A survey of augmented reality," Presence: Teleoperators and Virtual Environments, vol. 6, no. 4, pp. 355-385, August 1997.

[2] P. E. Debevec, "Rendering Synthetic Objects into Real Scenes: Bridging Traditional and Image-based Graphics with Global Illumination and High Dynamic Range Photography," Proc. of SIGGRAPH 98, pp. 189-198, July, 1998.

[3] G. Drettakis, L. Robert, S. Bougnoux, "Interactive Common Illumination for Computer Augmented Reality" Proc. of 8th Eurographics Workshop on Rendering, pp. 45-57, 1997.

[4] O. Faugeras Three-Dimensional Computer Vision: A Geometric Viewpoint, MIT Press, 1993.

[5] A. Fournier, A. Gunawan, and C. Romanzin, "Common Illumination between Real and Computer Generated Scenes," Proc. of Graphics Interface '99, pp.254262, 1993.

[6] B. K. P. Horn, Robot Vision, The MIT Press, Cambridge, MA, 1986.

[7] S. K. Nayar, K. Ikeuchi, and T. Kanade, "Surface reflection: physical and geometrical perspectives," IEEE Trans. PAMI, vol. 13, no. 7, pp. 611-634, 1991.

[8] I. Sato, Y. Sato, and K. Ikeuchi, "Seamless integration of virtual objects into a real scene based on a real illumination distribution," Technical Report, Computer Vision Laboratory, Institute of Industrial Science, The University of Tokyo, IIS-CVL-98-101, January 1998.

[9] C.Tomasi and T. Kanade, "Shape and Motion from Image Streams under Orthography: a Factorization Method," International Journal of Computer Vision, vol. 9, no. 2, pp. 137-154, 1992.

[10] K. E. Torrance and E. M. Sparrow, "Theory for offspecular reflection from roughened surface," Journal of Optical Society of America, vol.57, pp.1105-1114, 1967.

[11] R. Tsai, "A Versatile Camera Calibration Technique for High Accuracy Machine Vision Metrology Using Off-theShelf TV Cameras and Lenses," IEEE Trans. $R \& A$, vol. 3, no. 4, pp. 323-344, 1987. 